\title{
Prototipo de caja de curado inicial para muestras de cilindros de concreto
}

\section{Prototype of the initial curing box for samples of concrete cylinders}

\author{
Juleidys Vergara ${ }^{l}$, María Gutiérrez $z^{l}$ Randy Hernández ${ }^{l}$, Carlos Pérez ${ }^{l}$, Francisco Cedeñ ${ }^{2 *}$ \\ ${ }^{I}$ Licenciatura en Ingeniería Civil, Centro Regional de Azuero, Universidad Tecnológica de Panamá \\ ${ }^{2}$ Facultad de Ingeniería Civil, Centro Regional de Azuero, Universidad Tecnológica de Panamá
}

Resumen El proceso de curado de cilindros de concreto debe estar regido por los estándares establecidos por la ASTM (American
Standards for Testing Materials) y la FHA (Federal Highway Administration). Actualmente varios laboratorios que ofrecen el
servicio de curado incumplen en ciertos aspectos. "Curado inicial: Inmediatamente después del moldeado y el acabado, las muestras
se deben almacenar durante un período de hasta 48 horas en un rango de temperatura de 16 a $27^{\circ} \mathrm{C}\left[60\right.$ a $\left.80^{\circ} \mathrm{F}\right]$ y en un ambiente
que impida la pérdida de humedad del espécimen" [1]. De acuerdo con la ASTM, es posible plantear diferentes procedimientos
siempre y cuando se satisfagan dichas observaciones. Nuestro proyecto pretende solucionar ciertos aspectos incumplidos en las
prácticas comunes en el muestreo de cilindros de concreto, mediante un prototipo para el curado inicial del concreto que permita
mantener los especímenes en un rango de temperatura aceptable y que permita llevar a cabo un buen curado de los cilindros, ya sea
en unas instalaciones fijas o en sitio. El prototipo consiste en el aislamiento de los cilindros de concreto en una caja de
almacenamiento conectada a una unidad de enfriamiento con celdas de peltier alimentado por una fuente y manejado por un
microcontrolador de temperatura. Buscando que el concreto durante su fraguado mantenga una temperatura adecuada para obtener
la resistencia de diseño, ya que "Diferentes autores han documentado una ligera tendencia a la disminución de la resistencia a
compresión a largo plazo cuando aumenta la temperatura de curado, además de la posibilidad de que se produzca microfisuración"[4].

Palabras clave Curado, concreto, humedad, restricciones, norma, resistencia, sistema de enfriamiento.

\begin{abstract}
The process of curing concrete cylinders must be governed by the standards established by the ASTM (American Standards for Testing Materials) and the FEA (Federal Highway Administration). Currently there are several laboratories that offer the curing service fail in certain aspects. [1] "Initial Curing: Immediately after molding and finishing, samples should be recorded for a period of 48 hours in a temperature range of 16 to $27^{\circ} \mathrm{C}\left[60\right.$ to $80^{\circ} \mathrm{F}$ ] and in an environment that prevents the loss of moisture of the specimens ". According to the ASTM, it is possible to raise different procedures as long as those observations are satisfied. Our project addresses the needs of the incubators in the common practices in the sampling of concrete cylinders, by means of a prototype for the initial curing of the concrete that allows keeping the specimens in an acceptable temperature range and that allows to carry out a good Cylinder curing service, either in fixed installations or on site. The prototype consists of the isolation of the concrete cylinders in a storage box connected to a cooling unit with pellet cells powered by a source and operated by a temperature microcontroller. For the resistance of the resistance to humidity, the difference in resistance to the temperature of its resistance that occurs microcracking. "[4]
\end{abstract}

Keywords Curing, concrete, humidity, restrictions, norm, resistance, cooling system.

* Corresponding author: franciscocedeno3@gmail.com

\section{Introducción}

"El concreto es una mezcla de arena, grava, roca triturada, $\mathrm{u}$ otros agregados unidos en una masa rocosa por medio de una pasta de cemento y agua. En ocasiones, uno o más aditivos se agregan para cambiar ciertas características del concreto, tales como la ductilidad, durabilidad y tiempo de fraguado. Al igual que la mayoría de los materiales pétreos, el concreto tiene una alta resistencia a la compresión y una muy baja resistencia a la tensión" [1].

El concreto juega un papel esencial en historia de la construcción. En un principio cuando el hombre inició a levantar edificaciones mediante materiales como la arcilla o pétreos, aparece como exigencia la formación de pastas que mantuvieran unidos los elementos y dieran lugar a estructuras estables. 
Existen aspectos con los que un buen concreto debe cumplir dependiendo a las especificaciones para las que será aplicado como la resistencia, durabilidad y manejabilidad. Es muy importante tomar en cuenta que una fase fundamental para obtener un concreto ideal es la fase de curado inicial.

Las pruebas estándares de medición de hormigón indicadas por la ASTM (American Standards for Testing Materials) establecen un rango de temperatura para las muestras de 16 a $27^{\circ} \mathrm{C}$ y para mezclas con resistencias superiores a $6000 \mathrm{psi}$, temperaturas entre 20 y $26^{\circ} \mathrm{C}$, especifica en la norma ASTM C31 / C31M-18a en el punto 10.1.2 de la misma, el curado inicial, "Inmediatamente después del moldeado y el acabado, las muestras se deben almacenar durante un período de hasta 48 horas en un rango de temperatura de 16 a $27^{\circ} \mathrm{C}$ [ 60 a $80^{\circ}$ $\mathrm{F}]$ y en un ambiente que impida la pérdida de humedad del espécimen. Para mezclas de concreto con una resistencia especificada de $40 \mathrm{MPa}$ [6000 psi] o superior, la temperatura de curado inicial debe estar entre 20 y $26^{\circ} \mathrm{C}$ [ 68 y $\left.78^{\circ} \mathrm{F}\right]$ " [1]. Estos parámetros deben ser verificados in situ. Al comprobar que las condiciones del concreto cumplen con los parámetros establecidos, nos encontramos que el costo de las pruebas es elevado. Este proyecto tiene como objetivo, regular la temperatura a la que serán sometidas las muestras y obtener una unidad transportable, aplicando los principios necesarios para lograr dichas condiciones a un costo más accesible.

El curado inicial a los cilindros de concreto, no solo lo recomienda ASTM (American Standards for Testing Materials), sino, que lo recomienda también la [3] FHA (Federal Highway Administration), como parte de las prácticas comunes en el muestreo de cilindros de concreto.

\section{Alternativas}

"Varios procedimientos se pueden usar durante el período de curado inicial para mantener las condiciones de humedad y temperatura especificadas. Se debe usar un procedimiento apropiado o combinación de procedimientos (Nota 7). Proteja todas las muestras de la luz solar directa y, si se usan, dispositivos de calefacción radiante. La temperatura de almacenamiento se controlará mediante el uso de dispositivos de calefacción y refrigeración, según sea necesario. Registre la temperatura usando un termómetro de máximo a mínimo. Si se usan moldes de cartón, proteja la superficie exterior de los moldes del contacto con arpillera húmeda u otras fuentes de agua.

Se puede crear un ambiente de humedad satisfactorio durante el curado inicial de las muestras mediante uno o más de los siguientes procedimientos: (1) sumergir inmediatamente las muestras moldeadas con tapas de plástico en agua saturada con hidróxido de calcio, (2) almacenar en cajas de madera o estructuras, (3) coloque en fosas de arena húmedas, (4) cubra con tapas de plástico removibles, (5) coloque dentro de bolsas de plástico, o (6) cubra con láminas de plástico o placas no absorbentes si se toman medidas para evitar el secado y la arpillera húmeda es utilizado dentro del recinto, pero se evita que la arpillera entre en contacto con las superficies de hormigón. Se puede controlar un entorno de temperatura satisfactorio durante el curado inicial de las muestras mediante uno o más de los siguientes procedimientos: (1) uso de ventilación, (2) uso de hielo, (3) uso de dispositivos de calefacción o enfriamiento controlados por termostato, o (4) uso de métodos de calentamiento tales como estufas o bombillas. Se pueden usar otros métodos adecuados siempre que se cumplan los requisitos que limitan la temperatura de almacenamiento de la muestra y la pérdida de humedad" [1].

\section{Materiales y Métodos}

La unidad de la caja de curado inicial del concreto está formada por cuatro componentes: sistema de enfriamiento, registro de datos de temperatura, poder o fuente y la cámara de almacenamiento de cilindros.

\subsection{Enfriamiento}

Consta de disipadores de calor de aluminio, cuatro celdas de peltier, cables eléctricos, caja de madera de $30 \times 30 \times 30 \mathrm{~cm}$, cuatro reglitas de madera de $1 \times 1 \times 30 \mathrm{~cm}$, cuatro ventiladores de computadoras, cola blanca, gel térmico, conectores eléctricos de 2", microcontrolador de temperatura, tornillos, tuercas, esquineras de acero.

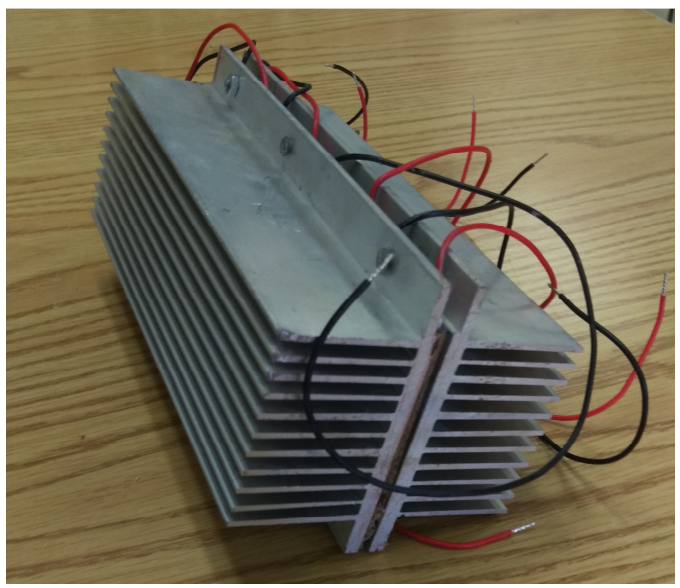

Figura 1. Unidad de enfriamiento.

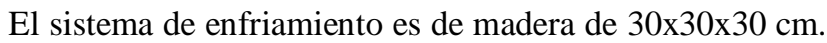
Esto se ubica en un compartimiento intermedio cerrado dentro de la caja de madera. En esta sección se colocan unidades de enfriamiento y extractores de calor. La unidad de enfriamiento está formada por dos disipadores de calor de aluminio y cuatro celdas de peltier unidas con gel térmico. Esto se ubica en un compartimiento intermedio cerrado dentro de la caja de 
madera. Los ventiladores y extractores son ubicados de manera que permitan conducir el aire frío del sistema de enfriamiento hacia la cámara de almacenamiento de cilindros y para expulsar el aire caliente del sistema de enfriamiento hacia el exterior o viceversa.

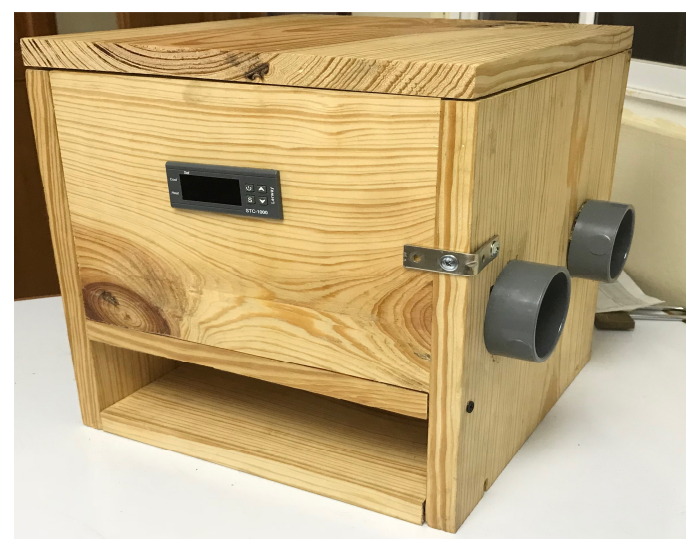

Figura 2. Prototipo de unidad de enfriamiento y microcontrolador de temperatura y encendido.

\subsection{Registro de datos de temperatura}

Consta de un dispositivo que mide la temperatura y la guarda en una memoria con capacidad para 16000 lecturas con un rango que puede variar entre $-40^{\circ} \mathrm{C}$ y $85^{\circ} \mathrm{C}$, para verificar que la misma se mantiene en el rango de valores especificados por la norma antes mencionada.

\subsection{Poder}

Consta de una batería de carga profunda "Las baterías de ciclo profundo son diferentes que las baterías automotrices plomo-acido. Las baterías de coche no deben ser utilizadas en sistemas de energías renovables. Las placas de una batería de automóvil son delgadas, lo que permite tener más placas dentro de una batería y por tanto más superficie de contacto. La extensa superficie permite una mayor descarga necesaria para arrancar un vehículo. La construcción de una batería de ciclo profundo se hace con placas más gruesas las cuales pueden retener la carga durante largos períodos de tiempo con una descarga continua y regulada" [2], con el equipo de registro de datos de temperatura se tomaron lecturas a un juego de 6 cilindros sin hacer ningún tipo de curado inicial para determinar aproximadamente el tiempo que deben trabajar los equipos de enfriamiento y así calcular el tamaño de la batería requerida, las cuales estuvieron por debajo de la temperatura máxima permitida alrededor de una hora y media según la gráfica 1 durante un periodo de medición de más de 25 horas.

\subsection{Almacenamiento de cilindros}

Materiales: carriolas, paredes de poliestireno recubiertas de aluminio, ángulos de acero, soldadura 6011. La misma contendrá las muestras cilíndricas de concreto aislándolas del ambiente exterior y ayudando a controlar la temperatura del aire que rodea a las muestras.

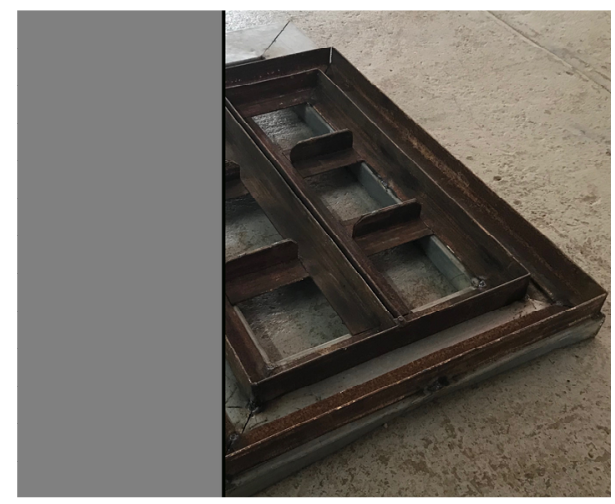

Figura 3. Base de la unidad de almacenamiento de cilindros.

En la figura 3 se muestra la base para almacenar los cilindros, con capacidad máxima para seis especímenes.

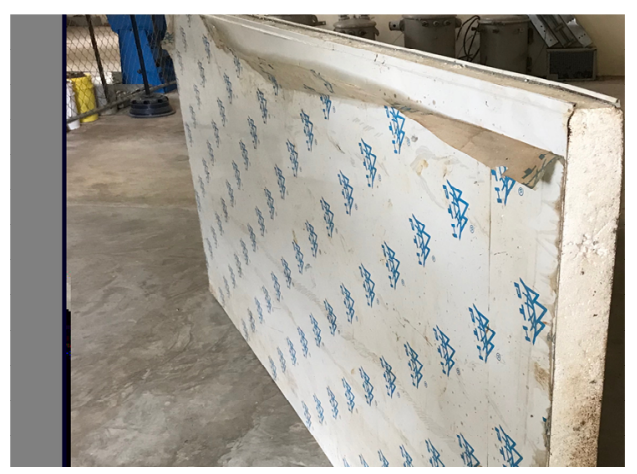

Figura 4. Paneles de poliestireno recubiertos de aluminios.

La figura 4 muestra los paneles de poliestireno para colocar como paredes de la cámara de almacenamiento.

\section{Resultados}

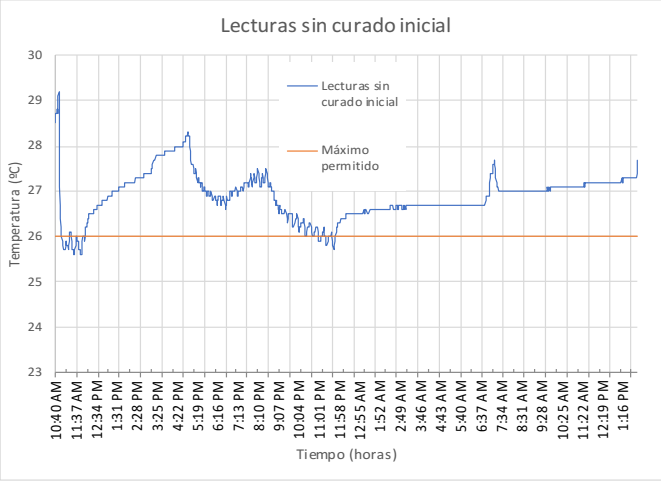

Gráfica 1. Lectura sin control de temperatura. 
La gráfica 1 muestra las lecturas de temperatura $\sin$ intervención de mecanismos que colaboren con el cumplimiento de la norma con respecto al rango de temperatura $\left(16\right.$ a $\left.27^{\circ} \mathrm{C}\right)$. Estas pruebas fueron realizadas en el Centro Regional de Azuero de la Universidad Tecnológica de Panamá y es muy notorio que en esta región las temperaturas se mantuvieron por un lapso de aproximadamente 24 horas, por encima de la temperatura máxima permitida por la ASTM (26$\left.27^{\circ} \mathrm{C}\right)$.

\section{Conclusiones}

- El impacto sobre la comunidad científica será significativo, debido a que se demostrará que en nuestro país se pueden hacer investigaciones para resolver problemas importantes. Se probó que el proyecto es viable a un bajo costo y que si se obtienen las patentes correspondientes se puede generar ingresos.

- Nuestro proyecto contribuye a cubrir una omisión a la norma de curado de los cilindros de concreto que tienen la mayoría de los laboratorios de ensayo de materiales de los países tropicales. Algunos de estos laboratorios no están cumpliendo en tener un control de la temperatura del aire alrededor de los cilindros mientras se lleva a cabo el curado inicial. El prototipo cumple teóricamente con las condiciones de trabajo especificadas por la norma, pero el tamaño, peso y materiales utilizados para la confección de este pueden ser mejorados. Para futuros trabajos podemos probar diferentes tipos de materiales, baterías o arreglos de los dispositivos usados en nuestro prototipo.

- Actualmente se intenta modificar aspectos en la unidad de enfriamiento que es la parte fundamental para que esta propuesta sea viable. Sin embargo, es necesario trabajar conjuntamente con especialistas de otras áreas como la ingeniería electromecánica y la ingeniería eléctrica para que aporten sus conocimientos al mejoramiento del proyecto.

\section{AGRADECIMIENTO}

"Agradecemos a Cecilio Solís y Cindy Santamaría por su asistencia técnica" y a Mijail Bernal por el apoyo financiero para la realización de este proyecto." Nota: agregar estos datos, que fueron extraídos para la competencia

\section{REFERENCIAS}

[1] American Standards for Testing Materials. (2018, Jun.). “ASTM C31 / C31M-18a”. [Online]. 5(10), pp. C31C31M.27953 Available:

https://compass.astm.org/download/C31C31M.27953.pdf [Jun. 12, 2018].

[2] Webosolar. (2018, Jun.). "bateria-ciclo-ciclado-profundo". [Online]. 1(1), pp. foro Available: http://www.webosolar.com/foro/bateria-ciclo-cicladoprofundo/ [Jun. 12, 2018].

[3] Federal Highway Administration Research and Technology. (2005, Feb.). "Guide for Curing of Portland Cement Concrete Pavements: Final Report". [Online]. 3(1), pp. FHWA-RD-02099. Available:

https://www.fhwa.dot.gov/publications/research/infrastructure /pavements/pccp/02099/chapt3.cfm [Mar. 8, 2016].

[4] Memoria de Trabajos de Difusión Científica y Técnica, núm. 10 (2012) "Influencia del curado en la resistencia a compresión del hormigón: estudio experimental” ISSN 1510-7450 • ISSN (en línea) 1688-9584. 